\title{
EVALUATION OF CARDIAC AUTONOMIC FUNCTION WITH HOLTER ECG MONITORING IN PATIENTS WITH SYSTEMIC SCLEROSIS
}

\author{
Lucian Muresan', Ana Petcu², Crina Muresan³, Cristina Pamfil', Mirela Rinzis², Laszlo Irsay ${ }^{4}$, \\ Ecaterina Bordnic ${ }^{4}$, Dana Pop ${ }^{1}$, Dumitru Zdrenghea ${ }^{1}$, Simona Rednic ${ }^{1}$ \\ ${ }^{1}$ Cardiology Department, Rehabilitation Hospital, Iuliu Hatieganu University of Medicine and Pharmacy, \\ Cluj-Napoca, Romania \\ ${ }^{2}$ Rheumatology Clinic, Iuliu Hatieganu University of Medicine and Pharmacy, Cluj-Napoca, Romania \\ ${ }^{3}$ Department of Internal Medicine, Second Medical Clinic, \\ Iuliu Hatieganu University of Medicine and Pharmacy, Cluj-Napoca, Romania \\ ${ }^{4}$ Physical and Rehabilitation Medicine Department, Rehabilitation Hospital, \\ Iuliu Hatieganu University of Medicine and Pharmacy, Cluj-Napoca, Romania
}

\begin{abstract}
Purpose. Cardiac autonomic dysfunction is frequently encountered among patients with systemic sclerosis. Its presence correlates with potentially fatal ventricular arrhythmias, having a good positive predictive value for mortality. The aim of this paper was to identify cardiac autonomic dysfunction in patients with systemic sclerosis using Holter ECG monitoring and to assess the possible correlations between its presence and other disease characteristics.

Material and methods. Forty nine patients with diffuse cutaneous and limited cutaneous scleroderma, diagnosed according to the American College of Rheumatology/EULAR 2013 criteria underwent ECG, transthoracic echocardiography, blood sample testing, chest X-ray and spirometry. Subsequently, all patients and a control group of 49 healthy subjects underwent Holter ECG monitoring with time and frequency domain heart rate variability (HRV) analysis.

Results. Scleroderma patients had significantly lower HRV values compared to controls: SDNN (123 \pm 39.4 vs. $143.2 \pm 32, p=0.001)$, SDANN $(137.2 \pm 34.9$ vs. $127.9 \pm 25.7, p=0.001), \mathrm{Tl}(-)(30.6 \pm 9.6$ vs. $38.1 \pm 8.9, p=0.01)$ and TINN ( $758.8 \pm 208$ vs. $815.1 \pm 138, p=0.04)$. The LF/HF ratio was significantly higher among patients with diffuse scleroderma $(1.18 \pm 0.34$ vs. $1.08 \pm 0.43, p=0.005)$. There was a positive correlation between the TI values, SDANN and the duration from the onset of Raynaud's phenomenon $(r=0.399, p=0.016)(r=0.419, p=0.011)$, between the SDANN values and systolic pulmonary arterial pressure (SPAP) values $(0.032, p=0.034)$ and a negative correlation between the LF/HF values and the patients' age $(r=-0.442, p=0.001)$, the duration from the onset of non-Raynaud's phenomenon ( $r=-0.395, p=0.034)$, echocardiographic value of $s P A P(r=-0.330, p=0.035)$ and the total number of premature ventricular contractions $(r=-0.0459, p=0.001)$.

Conclusion. Patients with systemic sclerosis often have cardiac autonomic dysfunction, which can be diagnosed with Holter ECG monitoring.
\end{abstract}

Keywords: systemic sclerosis, heart rate variability, Holter ECG

\section{INTRODUCTION}

Scleroderma is a systemic connective tissue disease of unknown etiology characterized by excessive fibrosis of the skin, blood vessels and internal organs, including the heart. Cardiac involvement is frequently encountered among scleroderma patients, with a prevalence of clinical manifestations of 15$35 \%$ (1). Subclinical cardiac involvement is even more frequent (2), and cardiac autonomic dysfunction is an important part of it (3). This can be present from an early stage of the disease, preceding the onset of fibrosis (4), and is characterized by a reduction

\footnotetext{
Correspondence address:

Lucian Muresan, Rehabilitation Hospital, Cardiology Department, 46-50 Viilor Street, 400347 Cluj-Napoca, Cluj, Romania

E-mail: lmure_san@yahoo.com
} 
in the parasympathetic tone and an increase in the sympathetic tone, especially in the early stages (5). The diagnosis of cardiac autonomic dysfunction is frequently established in a late stage of the disease, due to the discrepancies between the mild clinical manifestations and the severity of cardiac involvement.

Previous studies that assessed cardiac autonomic function in scleroderma patients have shown that the presence of cardiac autonomic dysfunction correlates with the presence of potentially malignant ventricular arrhythmias (6-8), having a good positive predictive value for mortality (9). Therefore, an early diagnosis of cardiac autonomic dysfunction is crucial for guiding optimal treatment from an early stage of the disease.

One of the existing methods for the evaluation of cardiac autonomic function is the study of heart rate variability (HRV). The variations in heart rate are mediated by the cardiac autonomic nervous system; therefore the analysis of HRV will reflect the influences of the autonomic nervous system upon the heart. A widely available, cost-effective, non-invasive method capable of identifying alterations in HRV is Holter ECG monitoring (10). Currently, there are only a few studies in the literature that evaluated HRV with Holter ECG monitoring for the assessment of cardiac autonomic function in patients with scleroderma $(7,9$, 11-18). Although these studies suggested a correlation between alterations in HRV, heart rate turbulence and the presence of malignant ventricular arrhythmias, they were conducted in small sample-sized populations (between 12 and 68 patients, with an average of 36 patients); therefore, additional studies are needed to confirm their main findings.

The aim of this paper was to identify the presence of cardiac autonomic dysfunction among scleroderma patients using Holter ECG monitoring and to assess the possible correlations between its presence and other characteristics of the disease.

\section{MATERIAL AND METHODS}

\section{Patient population}

Among the 134 patients, both men and women, diagnosed according to the American College of Rheumatology / EULAR 2013 criteria between November 2011 - July 2014 with either diffuse cutaneous or limited cutaneous scleroderma in the Rheumatology Clinic in Cluj-Napoca, 49 patients were included in the study. Exclusion criteria were: the presence of other rheumatologic diseases: rheumatoid arthritis, polymyositis, Sjogren's Syndrome, Still's disease, mixed connective tissue disease; cardiovascular diseases: ischemic heart disease, congestive heart failure, a left ventricular ejection fraction $<50 \%$, systemic arterial hypertension, moderate and severe pulmonary hypertension, significant valve disease, atrial fibrillation, atrial flutter, atrial tachycardia, major conduction disorders such as second and third degree AV block, complete left or right bundle branch block; the uses of antiarrhythmic drugs: beta blockers, class I and III antiarrhythmic drugs, Digoxin; other diseases that may impair HRV: diabetes mellitus, neuropathies, renal dysfunction (serum creatinine level of $>1.5 \mathrm{mg} / \mathrm{dl}$ ), significant pulmonary disease (severe obstructive or restrictive lung disease) and thyroid dysfunction (uncontrolled hyperthyroidism of hypothyroidism).

The control group comprised patients without known cardiovascular disease, admitted between April 2012 and June 2014 to the Physical and Rehabilitation Medicine Department, Rehabilitation Hospital, Cluj-Napoca. These patients were selected from a group of 85 patients who underwent Holter ECG monitoring for arrhythmia detection. Patients with arrhythmias detected on Holter ECG monitoring (1 patient with paroxysmal supraventricular tachycardia and 1 patient with paroxysmal atrial fibrillation) and / or associated cardiovascular diseases were excluded from the study. A total of 49 age and sex-matched subjects were then selected to represent the control group.

\section{Evaluation of patients}

\section{History taking and clinical exam}

Special attention was paid to the duration from the onset of Raynaud's phenomenon and of nonRaynaud's phenomenon, disease duration from the moment of diagnosis, the presence of symptoms: palpitations, exertional and resting dyspnea, vertigo, syncope; skin (Rodnan) score, telangiectasias, cutaneous calcinosis and digital ulcers.

\section{2 lead ECG and 24 hour Holter ECG monitoring}

The 12 lead ECGs were recorded using an Esaote P8000 electrocardiograph, with an ECG amplifier sensitivity of $10 \mathrm{~mm} / \mathrm{mV}$, at a speed of $25 \mathrm{~mm} / \mathrm{s}$. All ECGs were carefully screened for the presence of supraventricular or ventricular arrhythmias, conduction disorders, QRS axis deviations, signs of atrial or ventricular hypertrophy, myocardial ischemia, low QRS voltage, prolonged QRS and QTc duration. 
The 24 hour Holter ECG monitoring was performed using a 7-lead BTL CardioPoint H600 device, having a 2,000 $\mathrm{Hz}$ sampling frequency and 16 bits digital resolution. The studied parameters were: minimum, average and maximum heart rate while awake, asleep and during the whole 24 hour monitoring period; the presence of supraventricular arrhythmias: frequent premature atrial contractions (PAC), paroxysmal supraventricular tachycardias, atrial fibrillation, atrial flutter; the presence of ventricular arrhythmias: frequent premature ventricular complexes (PVC), non-sustained and sustained ventricular tachycardias; the presence of paroxysmal conduction disorders; QT and corrected QT interval. For HRV analysis, data was automatically processed by the integrated software and was subsequently verified and corrected when appropriate by a full time cardiologist in all cases.

\section{Echocardiography}

Transthoracic echocardiography was performed using an Esaote MyLab ${ }^{\mathrm{TM}} \mathrm{X}$-View 50 system, with a 7.5-10 MHz transducer. Standard image acquisitions were performed for all subjects. The assessed parameters were: chamber size and wall thickness, systolic function of the left ventricle (LV) and right ventricle (RV), diastolic function of the left ventricle, wall motion abnormalities, the presence of valve disease (stenosis and regurgitations), systolic, mean and diastolic pulmonary arterial pressure (sPAP, mPAP, dPAP) and the presence of pericardial effusion. Mild pulmonary hypertension was defined as sPAP of 35-49 mmHg, moderate pulmonary hypertension as SPAP between 50-69 $\mathrm{mmHg}$ and severe pulmonary hypertension as $\mathrm{SPAP} \geq 70 \mathrm{mmHg}$.

\section{Blood sample testing}

Blood sample testing included a complete blood count, erythrocyte sedimentation rate, glycemia, blood urea nitrogen, creatinine, electrolytes $\left(\mathrm{Na}^{+}\right.$, $\mathrm{K}^{+}, \mathrm{Ca}^{2+}, \mathrm{Cl}^{-}$), total cholesterol, HDL-cholesterol, LDL-cholesterol, triglycerides, uric acid, coagulation parameters (Quick time, INR, activated partial thromboplastin time), GOT, GPT, alkaline phosphatase, gamma-glutamyl transferase, bilirubin, total protein levels, albumin. Complement levels, C3, C4, circulating immune complexes, IgA, IgG, IgM, rheumatoid factor, antinuclear antibodies and antiScl 70 levels were also measured.

\section{Heart rate variability analysis}

Heart rate variability analysis was performed in both time and frequency domains.

\section{Time domain parameters}

The following parameters from the temporal domain were analyzed:

- NN, which corresponds to the number of RR intervals between 2 sinus QRS complexes

- HRV TI (Heart Rate Variability triangular Index), represents the integral of the density distribution (the number of all $\mathrm{NN}$ intervals) divided by the maximum of the density distribution. The measure is approximated by the value: (total number of $\mathrm{NN}$ intervals)/ (number of $\mathrm{NN}$ intervals in the modal bin)

- TINN (Triangular interpolation of the NN), which represents the base of the HRV TI triangle and approximates the distribution of the $\mathrm{NN}$ intervals. The HRV triangular index (HRV TI) represents the baseline width of the distribution measured as a base of a triangle, approximating the $\mathrm{NN}$ interval distribution (the minimum square difference is used to find such a triangle).

- SDNN (Standard Deviation of NN), corresponding to the standard deviation of the $\mathrm{NN}$ intervals (in milliseconds)

- SDANN (Standard Deviation of Averages of all Normal intervals), corresponding to the standard deviation of the average $\mathrm{NN}$ intervals in all 5-minute intervals of the entire recording.

- rMSSD (Root Mean Squared of Successive Differences), corresponding to the square root of the mean of the sum of the square differences between consecutive NN intervals

- NN50, corresponding to the number of differences between $2 \mathrm{NN}$ intervals $>50 \mathrm{~ms}$.

- pNN50, corresponding to the number of pairs of adjacent $\mathrm{NN}$ intervals differing by more than $50 \mathrm{~ms}$ in the entire recording, divided by the total number of all NN intervals.

\section{Frequency domain parameters}

The following parameters from the frequency domain were assessed:

- Low frequency: (LF), between 0.04 and $0.15 \mathrm{~Hz}$

- High frequency: (HF), between 0.15 and $0.4 \mathrm{~Hz}$.

In the present study, the LF/HF ratio was used.

Statistical analysis

Statistical analysis was performed using SPSS Statistics 20 (IBM. Chicago, Illinois). Descriptive statistics was used to summarize the characteristics of patients. Normality was assessed by using a Shapiro-Wilk test. Results are expressed as mean \pm standard deviation (SD) if normally distributed, or by 
median and interquartile range otherwise. Categorical variables are presented as counts and proportions $(\%)$.

Fisher's exact test was used to compare categorical variables. T-test for independent samples was used to compare normally distributed scalar variables and the Mann-Whitney-Wilcoxon test were used to compare non-normally distributed scalar values. Spearman's correlation coefficients were used to assess the relationship between the heart rate variability parameters and varied clinical and para-clinical patients' characteristics.

A $p$ value of $<0.05$ was considered statistically significant.

\section{RESULTS}

\section{General characteristics of the patients}

The main characteristics of the patients included in the present study are presented in Table 1.

There were no statistically significant differences between the diffuse cutaneous and limited cutaneous subgroups of scleroderma patients in what concerns the main clinical characteristics, with the exception of the skin score (15.2 vs. $11.1, \mathrm{p}<0.01)$ and the presence of anti SCL-70 antibodies ( 15 vs. 5, $\mathrm{p}<0.01$ ).

All patients were in sinus rhythm at the moment of the 12 lead ECG recording. No patient presented ST-T modifications suggestive of myocardial ischemia. There were no statistically significant differences in what concerns heart rate $(73.9 \pm 13.2$ vs. 73 $\pm 11, \mathrm{p}=0.8)$, QT interval ( $365.6 \pm 30$ vs. $365 \pm 30.5$, $\mathrm{p}=0.94)$ and QTc $(403.9 \pm 20$ vs. $400 \pm 26.8, \mathrm{p}=0.57)$ between the 2 subgroups of scleroderma patients.

On Holter ECG monitoring, scleroderma patients had significantly higher values of the minimum and average heart rate compared to controls (Table 2). The characteristics of supraventricular and ventricular arrhythmias were similar between the 2 groups of patients. There were no statistically significant differences between the maximum, minimum and average heart rate, total number of PAC, PVC, QT and QTc interval between patients with diffuse cutaneous and limited cutaneous scleroderma.

On transthoracic echocardiography, the main finding was the presence of diastolic dysfunction of the left ventricle $(25 \%$ of patients from the diffuse scleroderma subgroup vs. $36 \%$ of patients from the

TABLE 1. General characteristics of the patients

\begin{tabular}{|c|c|c|c|c|}
\hline Patients' characteristics & $\begin{array}{l}\text { Diffuse cutaneous } \\
\text { scleroderma }\end{array}$ & $\begin{array}{l}\text { Limited cutaneous } \\
\text { scleroderma }\end{array}$ & Total & $\mathbf{P}$ \\
\hline Number of patients $\mathrm{n}(\%)$ & $24(48.9)$ & $25(51.1)$ & $49(100)$ & - \\
\hline Sex, women $\mathrm{n}(\%)$ & $22(91.6)$ & $24(96)$ & $46(93.8)$ & 0.6 \\
\hline Average age (years) $n \pm$ dev std & $46.9 \pm 12.4$ & $52.1 \pm 11$ & $49.6 \pm 11.9$ & 0.13 \\
\hline $\begin{array}{l}\text { Disease characteristics } \\
\text { Rodnan score, average (extreme values) } \\
\text { Onset of Raynaud's phenomenon (years) } \\
\text { (average } \pm \text { std dev) } \\
\text { Onset of non-Raynaud's phenomenon (years) } \\
\text { (average } \pm \text { std dev) } \\
\text { Auto-antibodies, } n(\%) \\
\text { ANA negative } \\
\text { Anti SCL-70 positive }\end{array}$ & $\begin{array}{c}15.2(3-35) \\
9.1 \pm 9.6 \\
7.3 \pm 7.6 \\
2(8.3) \\
15(62.5)\end{array}$ & $\begin{array}{c}6.67(1-21) \\
11.8 \pm 8.3 \\
7.7 \pm 5.2 \\
1(4) \\
5(20)\end{array}$ & $\begin{array}{c}11.1(1-35) \\
10.3 \pm 9 \\
7.4 \pm 6.6 \\
3(6.1) \\
20(40.8)\end{array}$ & $\begin{array}{c}0.00092 \\
0.1 \\
0.43 \\
- \\
\mathbf{0 . 0 0 3}\end{array}$ \\
\hline $\begin{array}{l}\text { Associated conditions: } \mathbf{n}(\%) \\
\text { Mild pulmonary fibrosis } \\
\text { Mild/moderate respiratory dysfunction } \\
\text { Obstructive Pattern } \\
\text { Restrictive Pattern } \\
\text { Dyslipidemia }\end{array}$ & $\begin{array}{l}16(66.6) \\
7(29.1) \\
4(16.6) \\
9(37.5)\end{array}$ & $\begin{array}{l}12(48) \\
7(28) \\
3(12) \\
8(32)\end{array}$ & $\begin{array}{c}28(57.1) \\
14(28.5) \\
7(14.2) \\
17(34.6)\end{array}$ & $\begin{array}{c}0.25 \\
1.0 \\
0.7 \\
0.76\end{array}$ \\
\hline $\begin{array}{l}\text { Left ventricular ejection fraction (\%) } \\
\text { Average } \pm \text { std dev }\end{array}$ & $61.8 \pm 5.6$ & $62.7 \pm 6.4$ & $62.2 \pm 6.0$ & 0.58 \\
\hline $\begin{array}{l}\text { Left ventricular diastolic dysfunction: } \mathbf{n}(\%) \\
\text { Impaired relaxation } \\
\text { Pseudonormal } \\
\text { Restrictive filling }\end{array}$ & $\begin{array}{l}6(25) \\
6(25) \\
0(0) \\
0(0)\end{array}$ & $\begin{array}{l}9(36) \\
9(36) \\
0(0) \\
0(0)\end{array}$ & $\begin{array}{c}15(30.6) \\
15(30.6) \\
0(0) \\
0(0)\end{array}$ & $\begin{array}{c}0.53 \\
0.53 \\
- \\
-\end{array}$ \\
\hline $\begin{array}{l}\text { Medication: } \mathbf{n}(\%) \\
\text { ACE Inhibitors / ARBs } \\
\text { Calcium channel blockers }\end{array}$ & $\begin{array}{c}3(12.5) \\
6(25)\end{array}$ & $\begin{array}{l}2(8) \\
6(24)\end{array}$ & $\begin{array}{c}5(10.2) \\
12(24.4)\end{array}$ & - \\
\hline
\end{tabular}

ANA = anti nuclear antibodies; anti SCL-70 = anti SCL-70 antibodies; ACE Inhibitors = Angiotensin converting enzyme inhibitors; ARBs = Angiotensin II Receptor Blockers 
TABLE 2. The main findings of the 24-hour Holter ECG monitoring in scleroderma patients vs. controls

\begin{tabular}{|c|c|c|c|}
\hline & Scleroderma $(n=49)$ & Controls $(n=49)$ & $\mathbf{P}$ \\
\hline Maximum heart rate, $\mathrm{n} \pm$ std dev & $136.5 \pm 19$ & $129.5 \pm 20.6$ & 0.08 \\
\hline Average heart rate, $\mathrm{n} \pm$ std dev & $57.2 \pm 8.4$ & $49.8 \pm 6.2$ & 0.00001 \\
\hline Minimum heart rate, $\mathrm{n} \pm$ std dev & $78.9 \pm 10$ & $88.8 \pm 13.5$ & 0.00009 \\
\hline Average heart rate while awake, $n \pm$ std dev & $83.4 \pm 10.6$ & $75.8 \pm 10.2$ & 0.003 \\
\hline Average heart rate wile asleep, $n \pm$ std dev & $70.4 \pm 9$ & $63.2 \pm 8.5$ & 0.001 \\
\hline $\begin{array}{l}\text { Supraventricular arrhythmias } \\
-\geq 100 \text { PAC / } 24 \text { hours } \\
\text { - Runs of PAC } \\
\text { - Atrial tachycardia }\end{array}$ & $\begin{array}{l}10(20.4) \\
13(26.5) \\
1(2)\end{array}$ & $\begin{array}{c}10(20.4) \\
9(18.3) \\
2(4)\end{array}$ & $\begin{array}{c}1 \\
0.46 \\
-\end{array}$ \\
\hline $\begin{array}{l}\text { Ventricular arrhythmias } \\
\text { - } \geq 100 \text { PVC / } 24 \text { hours } \\
\text { - Systematized PVC (Bigemini, Trigemini) } \\
\text { - Couplets } \\
\text { - Non-sustained VT }\end{array}$ & $\begin{array}{l}11(22.4) \\
5(10.2) \\
4(8.1) \\
2(4)\end{array}$ & $\begin{array}{c}12(24.4) \\
6(12.2) \\
4(8.1) \\
0(0)\end{array}$ & $\begin{array}{l}1 \\
1 \\
1 \\
-\end{array}$ \\
\hline
\end{tabular}

$P A C=$ Premature atrial contractions $; P V C=$ Premature Ventricular Contractions $;$ VT = Ventricular Tachycardia.

limited cutaneous subgroup, $\mathrm{p}=\mathrm{ns})$. The systolic function of the left ventricle was normal in both subgroups of scleroderma patients. There were no statistically significant differences in what concerns the presence of mild valve disease and pulmonary arterial hypertension between the 2 subgroups of scleroderma patients.

\section{Cardiac autonomic function parameters}

Among scleroderma patients, there was a significant reduction in the values of the following time domain HRV parameters compared to controls: SDNN, SDANN, TI (-) and TINN. The rest of the time domain HRV parameters had similar values in both groups. There were no statistically significant differences in the frequency domain parameters between the 2 groups of patients (Table 3 ).

TABLE 3. Time and frequency domain heart rate variability parameters in scleroderma patients vs. controls

\begin{tabular}{|l|c|c|c|}
\hline & Scleroderma & Controls & $\mathbf{p}$ \\
\hline SDNN (ms) & $123 \pm 39.4$ & $143.2 \pm 32$ & $\mathbf{0 . 0 0 1}$ \\
\hline SDANN (ms) & $137.2 \pm 34.9$ & $127.9 \pm 25.7$ & $\mathbf{0 . 0 0 1}$ \\
\hline rMSSD (ms) & $43.4 \pm 44.9$ & $34.9 \pm 28.6$ & 0.54 \\
\hline TI (-) & $30.6 \pm 9.6$ & $38.1 \pm 8.9$ & $\mathbf{0 . 0 0 1}$ \\
\hline TINN & $758.8 \pm 208$ & $815.1 \pm 138$ & $\mathbf{0 . 0 4}$ \\
\hline NN(50)(-) & $5947.2 \pm 6205.6$ & $5736.5 \pm 5377.3$ & 0.95 \\
\hline pNN50(\%) & $6.3 \pm 6.3$ & $6.4 \pm 5.6$ & 0.61 \\
\hline LF/HF & $1.1 \pm 0.4$ & $1 \pm 0.3$ & 0.33 \\
\hline
\end{tabular}

SDNN = Standard Deviation of NN; SDANN = Standard Deviation of Averages of all Normal intervals; $r M S S D=$ Root Mean Squared of Successive Differences; $\mathrm{TI}=$ Triangular index; $T I N N=$ Triangular interpolation of the $\mathrm{NN} ; \mathrm{NN50}=$ the number of differences between $2 \mathrm{NN}$ intervals $>50 \mathrm{~ms}$; pNN50 = the number of pairs of adjacent NN intervals differing by more than $50 \mathrm{~ms}$ in the entire recording, divided by the total number of all NN intervals. $L F=$ Low frequency, between 0.04 and $0.15 \mathrm{~Hz}$; HF = High frequency, between 0.15 and $0.4 \mathrm{~Hz}$. See text for discussion.
When comparing HRV parameter values between patients with diffuse and limited cutaneous scleroderma, the only significant difference was in the LF/ HF ratio, patients with the diffuse form of the disease having significantly higher values $(1.18 \pm 0.34$ vs. $1.08 \pm 0.43, \mathrm{p}=0.005$ ) (Table 4$)$.

TABLE 4. Time and frequency domain heart rate variability parameters in patients with diffuse cutaneous scleroderma vs. limited cutaneous scleroderma

\begin{tabular}{|l|c|c|c|}
\hline & $\begin{array}{c}\text { Diffuse cutaneous } \\
\text { scleroderma }\end{array}$ & $\begin{array}{c}\text { Limited cutaneous } \\
\text { scleroderma }\end{array}$ & $\mathbf{p}$ \\
\hline SDNN (ms) & $121.1 \pm 46.4$ & $121.4 \pm 40$ & 0.45 \\
\hline SDANN (ms) & $101.8 \pm 37.1$ & $105.7 \pm 35.5$ & 0.26 \\
\hline rMSSD (ms) & $44.5 \pm 60$ & $43.7 \pm 44$ & 0.24 \\
\hline TI (-) & $31.3 \pm 10.6$ & $30.2 \pm 9.8$ & 0.94 \\
\hline TINN & $744.3 \pm 243.9$ & $748.2 \pm 216.3$ & 0.38 \\
\hline NN(50)(-) & $4441.4 \pm 4057.7$ & $5879.3 \pm 6087$ & 0.14 \\
\hline pNN50(\%) & $5.2 \pm 4.8$ & $6.3 \pm 6.2$ & 0.36 \\
\hline LF/HF & $1.18 \pm 0.34$ & $1.08 \pm 0.43$ & $\mathbf{0 . 0 0 5}$ \\
\hline
\end{tabular}

$S D N N=$ Standard Deviation of NN; SDANN = Standard Deviation of Averages of all Normal intervals; rMSSD = Root Mean Squared of Successive Differences; $T I=$ triangular index; TINN = Triangular interpolation of the NN; NN50 = the number of differences between $2 \mathrm{NN}$ intervals $>50 \mathrm{~ms} ;$ pNN50 = the number of pairs of adjacent $N N$ intervals differing by more than $50 \mathrm{~ms}$ in the entire recording, divided by the total number of all NN intervals. $L F=$ Low frequency, between 0.04 and 0.15 $\mathrm{Hz} ; \mathrm{HF}=$ High frequency, between 0.15 and $0.4 \mathrm{~Hz}$. See text for discussion.

Among all scleroderma patients, there was a positive correlation between certain time and frequency domain HRV parameters and the duration from the onset of Raynaud's and non-Rauynaud's phenomenon, the value of sPAP assessed by echocardiography, and a negative correlation between HRV parameters and the total number of PVC on Holter ECG monitoring (Table 5). When separated according to the scleroderma subtype, a negative correlation be- 
tween time domain HRV parameters and the ANA titer was found among patients with limited cutaneous scleroderma (Table 5).

TABLE 5. Correlations between time and frequency domain HRV parameters and clinical, echocardiographic and Holter ECG characteristics among scleroderma patients

\begin{tabular}{|l|l|c|c|}
\hline $\begin{array}{l}\text { HRV } \\
\text { parameter }\end{array}$ & $\begin{array}{l}\text { Clinical, echocardiographic } \\
\text { and Holter ECG } \\
\text { characteristics }\end{array}$ & $\begin{array}{l}\text { Correlation } \\
\text { coefficient r }\end{array}$ & P \\
\hline Diffuse and limited scleroderma & \multicolumn{2}{|l|}{} \\
\hline TI & Raynaud's phenomenon & 0.399 & 0.016 \\
\hline SDANN & Raynaud's phenomenon & 0.419 & 0.034 \\
\hline SDANN & Spap & 0.332 & 0.001 \\
\hline LF/HF & Age & -0.442 & 0.034 \\
\hline LF/HF & Non-Raynaud's phenomenon & -0.395 & 0.035 \\
\hline LF/HF & SPAP & -0.330 & 0.001 \\
\hline LF/HF & Total number of PVC & -0.459 & \\
\hline Diffuse scleroderma & \multicolumn{2}{|l}{} \\
\hline TI & Total number of PVC & -0.411 & 0.046 \\
\hline SDANN & Age & 0.411 & 0.046 \\
\hline SDANN & Raynaud's phenomenon & 0.485 & 0.03 \\
\hline SDANN & Non-Raynaud's phenomenon & 0.564 & 0.023 \\
\hline SDANN & Total number of PVC & -0.405 & 0.05 \\
\hline LF/HF & Non-Raynaud's phenomenon & -0.541 & 0.031 \\
\hline Limited scleroderma & \multicolumn{2}{|l}{} \\
\hline SDNN & ANA titer & -0.555 & 0.009 \\
\hline TI & Raynaud's phenomenon & 0.553 & 0.026 \\
\hline TINN & ANA titer & -0.523 & 0.015 \\
\hline TINN & Total number of PVC & -0.415 & 0.039 \\
\hline SDANN & ANA titer & -0.571 & 0.007 \\
\hline NN50 & ANA titer & -0.476 & 0.034 \\
\hline pNN50 & ANA titer & -0.516 & 0.017 \\
\hline LF/HF & Age & -0.651 & 0.0004 \\
\hline LF/HF & SPAP & -0.427 & 0.047 \\
\hline & \multicolumn{3}{|l}{} \\
\hline
\end{tabular}

$A N A=$ Antinuclear antibodies $; P V C=$ Premature ventricular contractions; $s P A P=$ systolic Pulmonary Arterial Pressure; $S D N N=$ Standard Deviation of NN; SDANN = Standard Deviation of Averages of all Normal intervals; $r M S S D=$ Root Mean Squared of Successive Differences; $T I N N=$ Triangular interpolation of the NN; NN50 = the number of differences between $2 \mathrm{NN}$ intervals $>50 \mathrm{~ms} ; \mathrm{pNN} 50=$ the number of pairs of adjacent $N N$ intervals differing by more than $50 \mathrm{~ms}$ in the entire recording, divided by the total number of all $N N$ intervals. $L F=L o w$ frequency, between 0.04 and $0.15 \mathrm{~Hz} ; \mathrm{HF}=$ High frequency, between 0.15 and $0.4 \mathrm{~Hz}$. See text for discussion.

\section{DISCUSSION}

The main finding of the present study is that cardiac autonomic dysfunction is present in scleroderma patients, and it can easily be diagnosed by assessing HRV using Holter ECG monitoring.

Heart rate variability is defined as subtle beat-tobeat variations / oscillations between 2 consecutive heart beats (19); it is controlled by the autonomic nervous system and reflects the degree of baroreceptors' sensitivity. Heart rate variability is impaired in scleroderma patients both in an upright and supine position, which was demonstrated with the use of tilt table testing (20). The lack of a significant increase in heart rate when switching from the supine to upright position reflects an altered baroreceptor modulation by the autonomic nervous system.

A reduction in HRV parameter values indicates an increase in the sympathetic influences and a decrease in the parasympathetic influences on the sino-atrial node, fostering electrical instability $(1,21)$. The main time domain HRV parameters are SDNN and HRV triangular index. These parameters assess heart rate variability on a global level and have a prognostic role. TINN also characterizes HRV on a global level. SDANN and rMSSD predict long-term alterations of HRV, and short-term, respectively; rMSSD and pNN50, which are parameters obtained by measuring differences of adjacent cardiac cycles can be considered as surrogate markers. They are specific markers of the parasympathetic nervous system and reflect the vagal tone, with rMSSD being more frequently used due to its superior statistical properties; SDNN and SDANNi reflect the circadian variations of HRV, being influenced by daily activities, short-term increase in sympathetic and parasympathetic tone. Regarding frequency domain HRV parameters, LF are considered to characterize the baroreflex control of the sympatho-vagal balance of arterial blood pressure and represent a marker of the sympathetic nervous system activity. HF characterizes sinus respiratory arrhythmia mediated by the parasympathetic nervous system. The LF/HF ratio is considered to characterize the sympatho-vagal balance.

The existence of a significant correlation between cardiac autonomic nervous system dysfunction and cardiovascular mortality is well known $(22,23)$. An imbalance in the sympatho-vagal nervous system results in an increased risk of potentially fatal ventricular arrhythmias development (24-28). Previous studies performed on post-myocardial infarction patients have shown that an imbalance of the autonomic nervous system represents an independent risk factor for malignant ventricular arrhythmias and sudden cardiac death (29-31). Other studies have demonstrated that HRV analysis can be used as a non-invasive diagnostic method for identifying the presence of autonomic neuropathy in diabetic patients (32).

In the present study, on Holter ECG monitoring, patients with scleroderma had significantly higher values of the average and minimal heart rate, both 
while awake and while asleep compared to controls. This observation, previously described by other authors (9), reflects an increase sympathetic tone in these patients. Studying HRV, Ferri et al. (9) assessed the cardiac autonomic function in a group of 30 scleroderma patients and found significantly higher heart rate values and lower HRV parameter values compared to controls. In addition, these parameters proved to have a good positive predictive value for mortality. The relative risk for mortality was increased among elderly scleroderma patients with positive anti Scl 70 antibodies. In the studies of Malliani et al. (33) and Appel et al. (34), the authors found an increased sympathetic tone in the studied subjects, manifested by higher heart rate values and higher serum levels of circulating catecholamines in patients with cardiac autonomic dysfunction.

In our study, scleroderma patients had lower values of certain HRV parameters, namely SDNN, SDANN, TI, TINN compared to controls, which reflects a cardiac sympatho-vagal imbalance. Such observations have previously been reported in other studies $(9,12-15,35,36)$.

The main finding of these studies was a decrease in time and frequency domain HRV parameter values and an increase in the LF/HF ratio. An increased $\mathrm{LF} / \mathrm{HF}$ ratio reflects an increased sympathetic tone in this population of patients. In our study, the LF/ HF ratio was increased only in patients with diffuse cutaneous scleroderma, compared to patients with limited cutaneous scleroderma.

Another important finding of this study is the correlation between certain HRV parameters and clinical and paraclinical characteristics of scleroderma patients. Among patients with both subtypes of scleroderma, there was a positive correlation between the value of TI and SDANN and the duration from the onset of Raynaud's phenomenon, between the SDANN values and systolic pulmonary arterial pressure (sPAP) values and a negative correlation between the LF/HF values and the patients' age, the duration from the onset of non-Raynaud's phenomenon, echocardiographic value of sPAP and the total number of premature ventricular contractions on Holter ECG monitoring. In the study of Othman et al. (13), the authors found a statistically significant positive correlation between certain HRV parameters and the presence of Raynaud's phenomenon, anti Scl 70 antibodies, as well as the skin score. Another study conducted by di Franco (12) analyzed the relationship between the alteration of HRV determined using Holter ECG monitoring and the presence of Raynaud's phenomenon assessed by capilaroscopy. The authors found a statistically significant correlation between an impaired HRV and the semiquantitative capilaroscopy score. They concluded that the coexistence of cardiac autonomic dysfunction with a more severe microvascular dysfunction could be a useful marker for the identification of patients with a higher mortality risk.

In the present study, in both groups of scleroderma subtypes there was a negative correlation between certain HRV parameters (TI, SDANN and TINN) and the total number of PVC on Holter ECG monitoring. Such a correlation has previously been described by Othman et al (13) and suggests the presence of a higher arrhythmic burden among scleroderma patients with more advanced autonomic dysfunction. These patients might have a worse prognosis compared to the other scleroderma patients, since the presence of cardiac autonomic dysfunction and numerous ventricular arrhythmias are associated with a higher risk of malignant ventricular arrhythmias (24-28).

Further studies are needed to prospectively evaluate the impact of cardiac autonomic dysfunction on cardiovascular morbidity and mortality among scleroderma patients.

\section{CONCLUSION}

Patients with systemic sclerosis have subclinical cardiac autonomic dysfunction, manifested by alterations in parameters characterizing heart rate variability, which can be diagnosed with Holter ECG monitoring. Given the correlation between cardiac autonomic dysfunction and the risk of malignant ventricular arrhythmia development, the Holter EGC monitoring may play an important role in identifying patients at risk for sudden cardiac death, allowing implementation of optimal therapeutic measures in an early stage of the disease. 


\section{REFERENCES}

1. Steen V.D. and T.A. Medsger, Jr., Severe organ involvement in systemic sclerosis with diffuse scleroderma. Arthritis Rheum, 2000. 43(11): p. 2437-44.

2. Candell-Riera, J., et al., Comprehensive noninvasive assessment of cardiac involvement in limited systemic sclerosis. Arthritis Rheum, 1996. 39(7): p. 1138-45.

3. Kahan A., G. Coghlan, V. McLaughlin, Cardiac complications of systemic sclerosis. Rheumatology, 2009. 48(Supplement 3): p. iii45-iii48.

4. Cozzolino, D., et al., Cardiac autonomic dysfunction precedes the development of fibrosis in patients with systemic sclerosis. Rheumatology (Oxford), 2002. 41(5): p. 586-8.

5. Dessein P.H., et al., Autonomic dysfunction in systemic sclerosis: sympathetic overactivity and instability. Am J Med, 1992. 93(2): p. 143-50.

6. Ferr, C., et al., Heart involvement and systemic sclerosis. Lupus, 2005. 14(9): p. 702-7.

7. Bienias P., et al., Heart Rate Turbulence Impairment and Ventricular Arrhythmias in Patients with Systemic Sclerosis. Pacing and Clinical Electrophysiology, 2010: p. no-no.

8. Wozniak J., et al., Evaluation of heart rhythm variability and arrhythmia in children with systemic and localized scleroderma. J Rheumatol, 2009. 36(1): p. 191-6.

9. Ferri C., et al., Autonomic dysfunction in systemic sclerosis: time and frequency domain 24 hour heart rate variability analysis. $\mathrm{Br} \mathrm{J}$ Rheumatol, 1997. 36(6): p. 669-76.

10. Kleiger R.E., et al., Stability over time of variables measuring heart rate variability in normal subjects. Am J Cardiol, 1991. 68(6): p. 626-30.

11. Bienias $\mathbf{P}$., et al., Heart rate turbulence assessment in systemic sclerosis: the role for the detection of cardiac autonomic nervous system dysfunction. Rheumatology (Oxford), 2010. 49(2): p. 35560.

12. Di Franco M., et al., Autonomic dysfunction and microvascular damage in systemic sclerosis. Clin Rheumatol, 2007. 26(8): p. 1278-83.

13. Othman K., A.N., Farouk H.M., Hassan I.M.A., Autonomic dysfunction predicts early cardiac affection in patients with systemic sclerosis. Clinical Medicine Insights: Arthritis and Musculoskeletal Disorders, 2010. 3: p. 43-54.

14. Ciftci O., et al., Cardiac repolarization abnormalities and increased sympathetic activity in scleroderma. J Natl Med Assoc, 2007. 99(3): p. 232-7.

15. Bielous-Wilk A., et al., Electrocardiographic evaluation in patients with systemic scleroderma and without clinically evident heart disease. Ann Noninvasive Electrocardiol, 2009. 14(3): p. 251-7.

16. Bienias P., et al., Pulmonary hypertension in systemic sclerosis determines cardiac autonomic dysfunction assessed by heart rate turbulence. Int J Cardiol, 2010. 141(3): p. 322-5.

17. Wranicz J.K., et al., (Evaluation of early cardiovascular involvement in patients with systemic sclerosis). Przegl Lek, 2000. 57(7-8): p. 389-92.

18. Morelli S., et al., Twenty-four hour heart period variability in systemic sclerosis. J Rheumatol, 1996. 23(4): p. 643-5.
19. Heart rate variability. Standards of measurement, physiological interpretation, and clinical use. Task Force of the European Society of Cardiology and the North American Society of Pacing and Electrophysiology. Eur Heart J, 1996. 17(3): p. 354-81.

20. Pancera P., et al., Autonomic nervous system dysfunction in sclerodermic and primary Raynaud's phenomenon. Clin Sci (Lond), 1999. 96(1): p. 49-57.

21. Deswal A., W.P. Follansbee, Cardiac involvement in scleroderma. Rheum Dis Clin North Am, 1996. 22(4): p. 841-60.

22. Lown B., R.L. Verrier, Neural activity and ventricular fibrillation. N Engl J Med, 1976. 294(21): p. 1165-70.

23. Schwartz P.J., M.T. La Rovere, and E. Vanoli, Autonomic nervous system and sudden cardiac death. Experimental basis and clinical observations for post-myocardial infarction risk stratification. Circulation, 1992. 85(1 Suppl): p. I77-91.

24. Shen M.J., D.P. Zipes, Role of the autonomic nervous system in modulating cardiac arrhythmias. Circ Res, 2014. 114(6): p. 1004-21.

25. Zipes D.P., Heart-brain interactions in cardiac arrhythmias: role of the autonomic nervous system. Cleve Clin J Med, 2008. 75 Suppl 2: p. S94-6.

26. Zipes D.P., Sympathetic stimulation and arrhythmias. N Engl J Med, 1991. 325(9): p. 656-7.

27. Zipes D.P., et al., Sudden cardiac death. Neural-cardiac interactions. Circulation, 1987. 76(1 Pt 2): p. I202-7.

28. Zipes D.P., et al., Influence of the autonomic nervous system on the genesis of cardiac arrhythmias. Pacing Clin Electrophysiol, 1983. 6(5 Pt 2): p. 1210-20.

29. Schmidt G., et al., Heart-rate turbulence after ventricular premature beats as a predictor of mortality after acute myocardial infarction. Lancet, 1999. 353(9162): p. 1390-6.

30. Bauer A., et al., Heart rate turbulence: standards of measurement, physiological interpretation, and clinical use: International Society for Holter and Noninvasive Electrophysiology Consensus. J Am Coll Cardiol, 2008. 52(17): p. 1353-65.

31. Wichterle D., et al., Predictive characteristics of holter-based postinfarction risk stratifiers appear superior to electrophysiological testing. Pacing Clin Electrophysiol, 2005. 28 Suppl 1: p. S182-6.

32. Balcioglu S., et al., Heart rate variability and heart rate turbulence in patients with type 2 diabetes mellitus with versus without cardiac autonomic neuropathy. Am J Cardiol, 2007. 100(5): p. 890-3.

33. Malliani A., et al., Cardiovascular neural regulation explored in the frequency domain. Circulation, 1991. 84(2): p. 482-92.

34. Appel M.L., et al., Beat to beat variability in cardiovascular variables: noise or music? J Am Coll Cardiol, 1989. 14(5): p. 1139-48.

35. Othman K.M., et al., Autonomic dysfunction predicts early cardiac affection in patients with systemic sclerosis. Clin Med Insights Arthritis Musculoskelet Disord, 2010. 3: p. 43-54.

36. Hermosillo A.G., et al., Autonomic dysfunction in diffuse scleroderma vs CREST: an assessment by computerized heart rate variability. J Rheumatol, 1994. 21(10): p. 1849-54. 